\title{
Sizing up Australia: The Next Step - summary
}

\author{
Daisy VEITCH ${ }^{\star a}$, Chris FITZGERALD ${ }^{b}$, Verna BLEWETT' ${ }^{c}$, Steve WARD ${ }^{d}$, Chang SHU ${ }^{e}$, \\ Kathleen ROBINETTE ${ }^{f}$ \\ ${ }^{a}$ SHARP Dummies Pty Ltd, Adelaide Australia; \\ ${ }^{\mathrm{b}}$ Risk \& Injury Management Services Pty Ltd, Melbourne, Australia; \\ ${ }^{c}$ Appleton Institute, Central Queensland University, Adelaide, Australia; \\ 'University of New South Wales, Sydney, Australia;

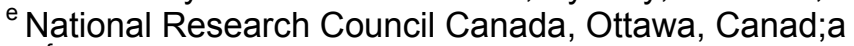 \\ ${ }^{f}$ Oklahoma State University, Oklahoma City, USA \\ http://dx.doi.org/10.15221/13.135
}

\begin{abstract}
This paper describes the development of the methods and scientific parameters for a proposed Australian Body Sizing Survey. It builds on a previous research that established the need for such a survey. The aim of this research was to learn from others' experience and establish methods for planning and conducting a robust national sizing survey that would meet the needs of a broad range of anticipated stakeholders. Two further stages are needed before an Australian Body Sizing Survey can be realised: 1) to identify stakeholders, test the survey design against their needs and finalise the method and scientific parameters and 2) to conduct the Australian Body Sizing Survey by collecting raw data according to the agreed method and scientific parameters.
\end{abstract}

In this research we sought answers to basic questions, such as: What should an anthropometric survey deliver? What components make it useful? Will it work? Will it be fit for purpose? The research established the characteristics of a good measurement with examples of the technologies that can produce the necessary quality, while being open to technologies that are under development and might be suitable in the future. We describe a process for sampling that is based upon error estimates from past studies and includes methods for estimating sample size and recruitment strategy. Accuracy, validity and cost-effectiveness are all important considerations and we propose methods that take into account time per subject, size and skill of the data collection team, and the number and type of measurements and demographic variables needed to meet stakeholder requirements.

The research examined: 1) factors influencing the budget and resources, 2) international and Australian academic and 'grey' literature about national sizing surveys and international standards in other leading countries, and 3) processes to define the scope of the Australian Body Sizing Survey. It addressed the key features of stakeholder engagement and how this would determine the range and type of measurements to be obtained. It also described the systems engineering model that would be used to develop the testing required to finalise the survey method, business plan and costing. It outlined possible sizing survey methods including recommended sampling methods, recruitment strategies and data management.

The research findings can be grouped into six key areas that are critical to the effective and efficient development of the Australian Body Sizing Survey. Firstly, 1-D and 3-D data types provide different information and both are essential for a high quality, useful survey. Secondly, new 4-D (high quality 3-D scans captured while the subject is in motion) could provide a cost effective way to capture fit information. Thirdly, it is critical that stakeholders be engaged early in the process. Fourthly, although ISO Standards provide basic templates for the development of a survey, they are not sufficient. Fifthly, a systems-engineering approach balancing technical factors, cost, time, and needs, is required, and lastly, the value propositions from industry demonstrated that a survey conducted in the right way will enable better design and safer and healthier work places and equipment.

Keywords: Engineering anthropometry, human factors, workplace design, product design, sizing survey, work health and safety, 3D body scanning

Acknowledgements: This research was funded by Safe Work Australia, an agency of the Commonwealth Government of Australia. 


\section{Introduction}

A report published in 2009, Sizing Up Australia - How Contemporary is the Anthropometric Data Australian Designers Use? demonstrated the gap between inadequate anthropometric data and the production of workable designs, workplaces or access to workplaces, and the need for a well-executed, comprehensive anthropometric survey representative of the Australian population (1). The principal driver of this research was the recognition that design plays an important role in producing healthy and safe working environments, and that anthropometric data can make an important contribution to the design process. This initial research stimulated the need to take the next small step towards conducting an Australian Body Sizing Survey and the results of this second phase of research are summarized here. A more comprehensive report of this second phase of study, titled, Australian Body Sizing Survey: Defining the method and scientific parameters, is in press (2). This research focused on the criteria for identifying and engaging partners/stakeholders and on developing a method to ensure the survey will provide them with:

- Data that will fulfill their needs,

- Subjects from their target population, and

- Measurements, postures, poses, landmarks, models etc. that they need.

It established the parameters needed to estimate the cost and the process(es) required to select measurements and subjects once the stakeholders are engaged in the work.

Although the needs of stakeholders and other users of the Australian Body Sizing Survey provide the foundation for the development of the method and scope of the sizing survey, other parameters need to be considered because they impact on cost, time needed, efficiency and the effectiveness of the survey. These include: the sampling strategy to ensure a reliable representation of the very diverse Australian population; the demographic data, the type of measurements and the fit variables, all of which have impact on the amount of time that each subject would be needed for the survey; and the size of the team of people needed to collect the sizing survey data.

Other considerations in developing the method for the Australian Body Sizing Survey are agreement on what constitutes a "good" measurement and the capacity of the various technologies to deliver a "good" measurement. These are crucial to ensuring that the Australian Body Sizing Survey contains data of sufficient quality to be useful to a wide range of designers and other stakeholders as well as ensuring that the Survey provides a strong foundation for the addition of data from future generations. The Australian Body Sizing Survey would not be produced in a vacuum. Some limited sizing surveys are available and it may be possible to use error estimates from these studies. Existing national census data may allow Australian sample size and locations to be matched with existing Australian Bureau of Statistics (ABS) data, such as height, weight, age, ethnicity and regional information, in order to propose a random, unbiased, or other subject recruitment method for which bias can be estimated. Given the importance of such a survey for the Australian population, we need the best assistance, including international expertise, to inform decision-making as part of an International Technical Committee of experts.

\section{Background}

The experience of other nations and researchers is important in informing the method that Australia might use in the design of an effective Australian Body Sizing Survey. That's why a literature review is important. There are different categories of use for anthropometry besides engineering or design anthropometry; public health, sports science, forensic and biological anthropology have been excluded from this research as it focuses purely on engineering or design anthropometry. The end use or purpose determines the suitability of each data set, and not all anthropometric data are suitable for engineering and design purposes (see (1) and (3)). Many existing datasets collected for these other purposes cannot meet the requirements of designers. Thus, identifying the stakeholders and knowing their end use during the planning stage is crucial in ensuring that the anthropometric data collected for the Australian Body Sizing Survey will be fit for purpose.

There are a number of not-for-profit organisations directly supporting this type of endeavour, such as AGARD - Advisory Group for Aerospace Research and Development (4), WEAR - World Engineering Anthropometry Resource (5), and CODATA - International Council for Science: Committee on Data for Science and Technology (6). WEAR has particular importance to the Australian Body Sizing Survey because it could potentially provide a clearing-house for the data that could make it readily accessible to stakeholders, as depicted in Figure 1. 


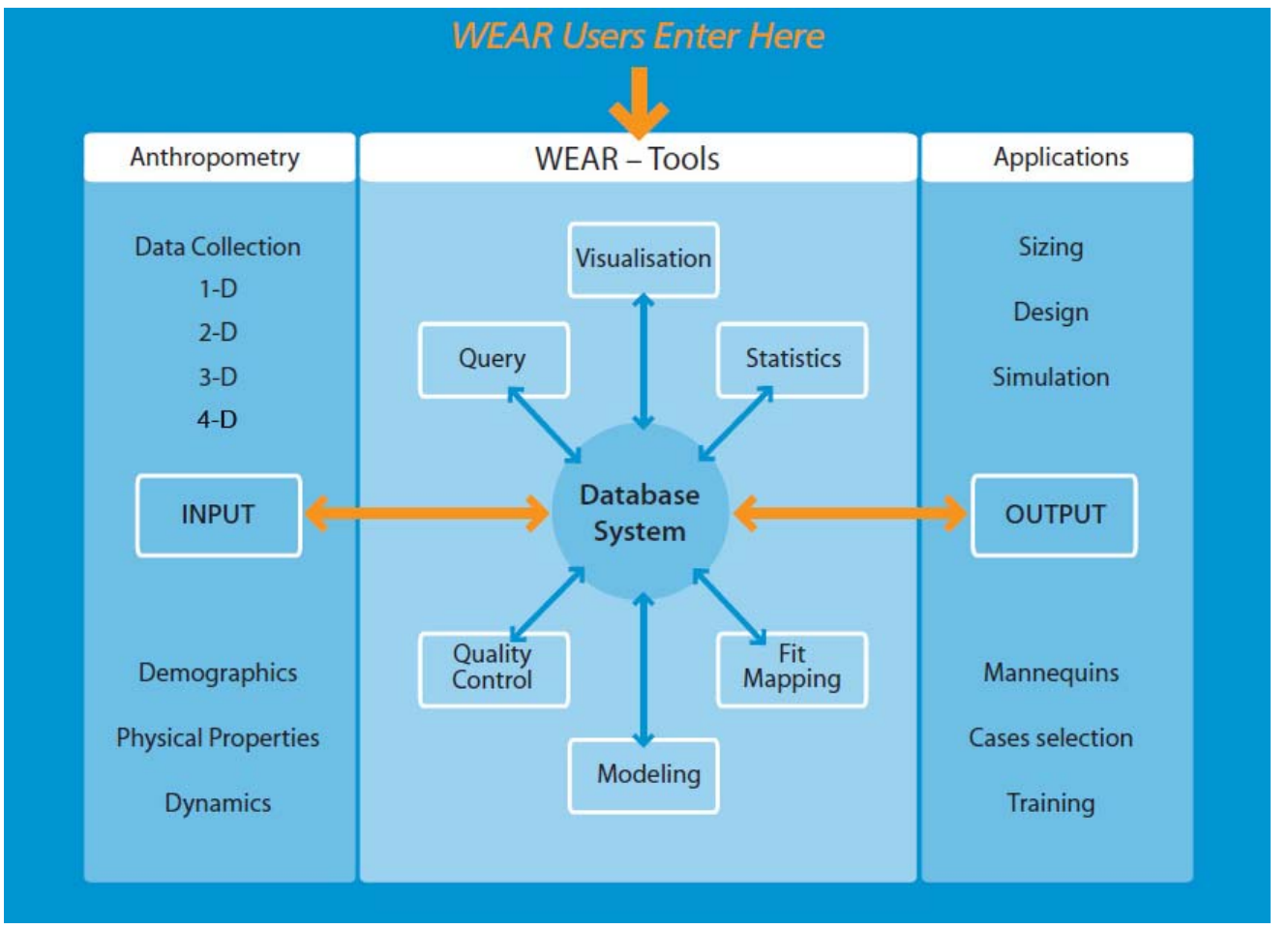

Figure 1: WEAR diagram to represent where Data Types fits into the scheme of things how Anthropometry interacts with Tools to create Applications.

\section{Anthropometry}

There are many ways to measure the human body and new tools are continuing to be developed, but there are four main measurement types for engineering anthropometry: one-dimensional (1-D), two dimensional (2-D), three-dimensional (3-D) and 3-D motion, sometimes called 4-D with the fourth dimension being time. 1-D and 3-D data provide different kinds of information yet both are essential for a high quality, useful survey. 2-D can be extracted effectively from 3-D as a sub-set and so it doesn't need separate consideration. Although claims are sometimes made (7), that 1-D measurements can be automatically and reliably extracted, i.e., without human intervention or viewing, from 3-D scans, the reality is that highly accurate 1-D measurements can be extracted from high quality 3-D scans (8) only with pre-marking the body and manual intervention and screening. Circumference measurements automatically extracted from 3-D scans are not usually the same as, and are therefore not comparable to, the traditional measurement taken on a live person (9). Fast, high quality 3-D scans acquired while the subject is in motion (4-D) can vastly increase the understanding of the influence that age, load, and apparel have on the capacity of people to live and work safely and effectively. 4-D scanning is just emerging as a cost-effective tool.

Different data types have different strengths and weaknesses. When weighing strengths and weaknesses it might be helpful to think of an analogy. Which is better - a hammer or a screwdriver or maybe a drill? If the goal is to put a nail in the wall clearly the hammer is best. If the goal were to put in a screw, the screwdriver would be best or even an electric drill. The tool needs to be fit for the task in hand. That is why it is crucial to plan carefully and ensure the data type is the best one for the purpose. Thus it is crucial that end users of data are clearly identified through stakeholder engagement.

All types of anthropometric data have advantages and disadvantages. An Australian survey should review the latest developments to weigh up strengths and weaknesses in data types and technology to deliver the most useful combinations for the stakeholders involved in the project within the practical limitations of the survey design. This approach will deliver an effective survey that represents value for money.

Through the exploration of the disadvantages of 1-D data, the missing link - the relationship between anthropometric data and design - is revealed. This essential link is the "fit" of products. 3-D data provide powerful information, but they are complex to analyse. 1-D is easy to use but insufficient, especially for complex design. For the best of both data types an Australian survey should collect both. 
Making data comparable is both necessary and problematic especially in the light of language, quality and measurement description differences. Ways to manage these problems are discussed in detail, such as using an Extensible Mark-up Language (XML) schema as a type of Rosetta stone (10).

A historical timeline has been created highlighting important developments in engineering anthropometry (2). It is vital to understand that developments build upon each other to create good solutions and there are good reasons why something has been done a certain way. This helps inform 'lessons learned' and draws on past experience to inform future good practice.

\section{Referential standards for sizing surveys}

Five relevant International Standards (ISO) exist (11-15). ISO Standards provide a basic template for the development of a survey but are not sufficient in themselves to define all aspects of the survey. ISO/TC 159 is in the process of developing New Working Items to create validation protocols missing from the Standards (16). In the meantime a future Australian survey must still be validated, so both lessons learned from past surveys and current tools, such as the Anthropometric Measurement Interface (AMI) (17), are essential material for supplementing the standard.

Different types of information such as demographic data including age (examination date minus birth date equals subject's age) are necessary to accompany body-size/shape data to relate the user populations to potential end-uses. ISO 15535 (13) describes the minimum recommended background information required and any survey conducted should comply in the interests of international comparability. A set of static measurement definitions for is provided in ISO 7250-1 and ISO 7250-2 reports on population data collected from a number of countries according to these definitions. 3-D scan extracted measurements are examined in detail in ISO 20685 with the intention of comparing measurement instruments (scan versus traditional) to determine whether they are close enough, under a certain set of conditions, to be considered comparable measurements. Methods are suggested to reduce error in 3-D scanning to help meet the above criteria.

Additional data requested by the stakeholders should also be considered.

Landmarks, their uses, traditional-style measurements, 3-D scanned data and other data related to standards, e.g., strength, are substantial considerations in the survey planning.

\section{Anthropometric surveys}

The type of measurements collected in an anthropometric survey makes some surveys more useful than others for engineering design. High quality in both 1-D and 3-D measurement types maximizes usefulness for design and engineering purposes. Rows 1 and 2 list some of the 120 surveys available through the WEAR data network. Surveys listed in Row 3 have known quality issues. Generally quality issues include taking the measurement in the wrong place on the body (18) and automated landmark locations that differ from those placed by an expert by $20 \mathrm{~mm}$; this is good enough for shape comparisons but not measurement extraction (19).

Table 1 - surveys in categories of usefulness.

\begin{tabular}{|l|l|l|}
\hline Type of measures & Comment & Example \\
\hline $\begin{array}{l}\text { 3-D scans }+1-D \text { measures } \\
\text { (1-D measures collected } \\
\text { traditionally) }\end{array}$ & $\begin{array}{l}\text { Most useful } \\
\text { Recommended by ISO } \\
\text { High Quality }\end{array}$ & $\begin{array}{l}\text { CAESAR (2002) } \\
\text { Size Korea }(2003,2010)) \\
\text { Size Japan }(2006) \\
\text { Some others... }\end{array}$ \\
\hline $\begin{array}{l}\text { 1-D measures only } \\
\text { (taken traditionally) }\end{array}$ & $\begin{array}{l}\text { Mostly military (bias towards } \\
\text { males) } \\
\text { High quality } \\
\text { No 3-D }\end{array}$ & $\begin{array}{l}\text { ANSUR }(1988) \\
\text { Size Korea }(1986,1992,1997) \\
\text { Many others.... }\end{array}$ \\
\hline $\begin{array}{l}\text { 1-D measures only } \\
\text { (taken with a 3-D scanner) }\end{array}$ & $\begin{array}{l}\text { Least useful } \\
\text { Documented quality issues } \\
\text { Often only summary statistics } \\
\text { Sometime 3-D scan discarded }\end{array}$ & $\begin{array}{l}\text { Size UK (2001) } \\
\text { Size USA (2003) } \\
\text { Spanish Size Survey (2007) } \\
\text { Size Germany (2009) } \\
\text { Alvanon }\end{array}$ \\
\hline
\end{tabular}




\section{How are anthropometric data used in industry?}

Data are used by industry according to their end-use, such as:

- worn things/apparel sizing;

- $\quad$ built environments - accommodation - workplace design and the built environment; and

- fit/accommodation mapping which includes digital human models and fit mapping.

Case studies included the now famous US Navy example that used fit mapping in US Navy women's uniform study(20).

Mellian, Ervin et al. conducted a fit-mapping exercise which resulted in reshaping recommendations that reduced the number of major alterations from $75 \%$ to less than $1 \%$. In addition it recommended dropping overlapping sizes and introducing missing sizes for different hip-waist ratios improving the accommodation rate of the women from $23 \%$ to $99 \%$, with the same number of sizes. Obviously this has a large financial implication; especially since the women had to pay for their own alterations.

Without fit-mapping the designer has access to limited information about the body's surface, that it, about shape and size, making it difficult to test for error or determine which of the multiple solutions is best.

Another outstanding example is the cockpit example where accommodation mapping was used to produce tools "including a body clearance/size requirement chart to identify the possible career track of a potential recruit based on their individual measurements... This information was then used to create a pilot career path tool identifying the body sizes and proportions that could safely operate the various aircraft in the career pipeline. Failure in any one of the accommodation criteria could make the pilot unsafe to fly an aircraft" $(21,22)$. Output is shown in Figure 2 with shows aircraft arranged in order of mandatory training left to right. Codes are $\mathrm{H}=$ helicopter, $\mathrm{T}=$ trainer, $\mathrm{F}=$ fighter jet, $\mathrm{C}=$ cargo plane. The number is the identity of the aircraft.

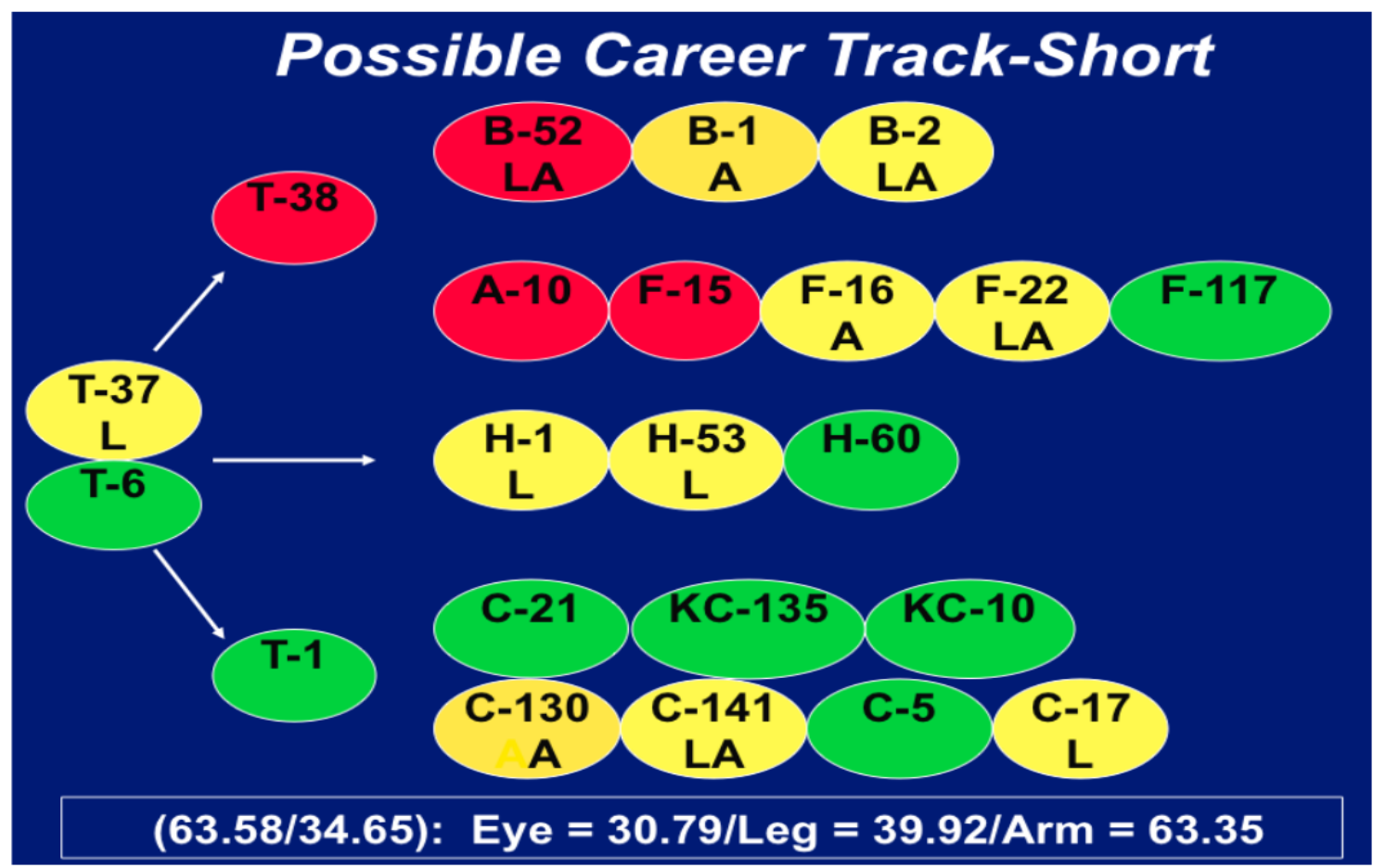

Figure 2: Red indicates this person is unsafe to fly, green is safe to fly and yellow means that person would need an additional assessment before being judged safe or unsafe to fly that plane. Source Zehner.

This is an example of a cost-effective tool designed to avoid accidents and improve future designs.

In addition good design requires raw data and product specific analysis. Raw data means the actual individual data must be available to combine or recombine depending on the need - not summary statistics. Product specific analysis means the relationship between the human and the system must be known and quantified for a particular product. 
Regarding digital human models - currently there is a technology gap in capturing accurate and detailed shape information while the subject is moving. However this gap might be closed soon.

The next generation of models that is under development will be able to accommodate shape data from real people but the models will only be as good as the input data. That is why a diverse range of high quality, raw 3-D, 1-D and physical landmarking/segmentation data is so important to the validity and hence usefulness of the tool. In addition, their usefulness depends on understanding and characterising the relationship between the models and the desired environment, including motion and encumbrance. It is not sufficient to know how they might be aligned - to have confidence the designer must be able to measure how they are aligned (4). This needs to be quantified by fit-mapping.

A survey conducted in the right way, although a complex task, will enable better design and safer and healthier work places and practices.

\section{Technical detail in methods - body sizing surveys}

The ISO standard 15535 delineates population sampling strategies and sample size (13). The CAESAR survey gives a specific and detailed example (23). The Australia method will require customization to the Australian context and discussion of some of the trade-offs (2).

Quality is a very important consideration for the Australian survey. The literature relating to quality of collecting traditional-style measurements is extensive and well documented. What is less well documented was the quality of the new or emerging technologies. However CAESAR had extensive and detailed documentation of additional quality systems such as, a system for determining the range of outliers based on previous surveys using a regression outlier analysis which beeped when data was entered into the computer to alert the investigator to any potential errors (23).

The purchase of a scanner (hardware) is another important consideration. At the moment there is no ISO standard to define the terminology and validation of 3-D scanner hardware and software although there is a New Working Group for ISO 159 assigned to develop this. In the absence of this a set of purchasing criteria was proposed by Kathleen Robinette to ASTM in 2005 (24).

\section{Lessons learnt from past surveys}

The literature focusing on lessons learned in relation to anthropometric sizing surveys was very hard to find. Only one self-reported paper could be located giving an honest appraisal of the sizing survey with suggestions of improvements. This was for the CAESAR survey (25). Other literature that was critical was in relation to quality problems with the data of other surveys and were not-self reported - rather reported by end-users of data experiencing problems. Interestingly there are no external reports of problems with CAESAR data, instead the successful industry applications have mostly come from the use of CAESAR data often in combination with the collection of additional fit/accommodation data (19, 26-36).

The CAESAR self-reflection revealed that, for a large and complex project collecting 3-D body scan data and traditional-style measurements for more than 4,000 subjects, in excess of 13,000 scans, using 2 different scanner types in 3 different countries, success stemmed from two main avenues. First, a systems engineering approach to the development, preparation and delivery is the groundwork for good planning and organization, and second, gaining early and ongoing feedback from stakeholders and those working within the project ensured the data collected for correct and fit for purpose (25).

The CAESAR project engaged stakeholders at the outset. These stakeholders focused on end-users of the data and they were given a significant capacity to define their needs and preferences and so ultimately define the scope of the project and determine the range of measures that would be obtained. This engagement was fundamental to the success of the project as demonstrated by the modification of the original plan from scanning only the traditional seated erect pose to adopt the industry stakeholders' preference for a natural seated pose.

The measurement process that needs to be conducted within a finite period for each subject installs an inherent limitation on the ability of the project to capture as much measured data as all stakeholders are likely to want (25). For example, in the CAESAR development and testing process, the time taken to obtain 90 landmarks was greater than an hour, a survey duration target identified when surveying prospective participants. This number of landmarks needed to be revised down to 72 so the survey period matched the one-hour window. 


\section{Conclusions and implications for an Australian Body Sizing Survey}

It is fortunate that in the development of the parameters for an Australian Body Sizing Survey there is an abundance of experience and supporting written reference material that can inform best practice.

An Australian sizing survey will seek to use a systems engineering approach to maximise effective development and management of the survey. Success would be defined by how useful the data are to solve engineering anthropometric problems - how fit for purpose the data are? The project would need to assemble a multidisciplinary team at the outset and adopt this approach for the planning and co-ordination of resources and logistics, working with stakeholders to determine which measurements to collect, and testing and finalising the measurement technologies and methods. Risk management principles would also be applied to establish effective management systems and methods to run the Australian Body Sizing Survey project.

With regards to quality criteria for measurement technologies and obtaining measurements there are currently no standards for 3-D body scanning technology, both hardware and software, and a buyer beware situation prevails. The Australian Body Sizing Survey will need to develop performance criteria for scanners and any scanners being considered for use within the survey would need to be tested against these criteria. It will not be sufficient to rely on manufacturer specifications and promotional material.

To manage quality over a period of time a pool of trained and competent assessors will be needed, as inevitably with a long and complex project there will be necessity for appropriate backup and support. Traditional measurements should comply with quality standards of inter- and intra-rater reliability.

A range of different stakeholders will be interested in different components of the survey, from raw data through tools and applications to end-users. However, early and ongoing engagement will be vital to ensure that the survey is run to achieve best outcomes for all stakeholders.

An ultimate goal of the Australian survey would be to maximise the possible measurement outputs from the raw 3-D scanned data, to minimise the number of traditional measurements that need to be taken and maximise the overall efficiency of the survey while optimising quality and accuracy.

A method of managing the technical aspects of the rationalization process will be to establish an International Technical Committee consisting of subject matter experts to act as an arbiter in decisions such as prioritisation and justification for measurements selected and consequently landmarks.

The combination of ISO standards and the recorded experiences of others provides detailed information about the range of data that can be collected and how. ISO standards also define baseline demographic data that could be included within the Australian survey so they correspond with other anthropometric databases that also used ISO baselines. However, the ISO standards as a single set of references, while necessary, are not sufficient.

Stakeholder involvement is essential to define the scope, final method and hence resources required for a survey that delivers stakeholders' needs. Once stakeholders have been engaged and consulted and a resulting list of measurements drawn up, a trial study needs to be conducted with the proposed hardware and software. This would be used to test accuracy and consistency in using 3-D scanned data, manual or automated measurement extraction and, when these cannot be obtained, traditional style measurements for some of these dimensions would be collected. Pilot testing should cover all aspects of the survey in line with a systems engineering approach.

The Australian Body Sizing Survey presents an opportunity to extend the experience of previous surveys by developing and using the most up to date survey method and using scanning technology to capture high quality raw data, possibly with the inclusion of 4-D for the first time.

Fit-mapping in a large scale National Sizing survey such as the Australian Body Sizing Survey would break new ground and would be the first instance of this work on a civilian population. The ability of designers to not just use high quality anthropometric data but to understand and potentially more accurately predict fit for their populations would be commercially very valuable, help drive innovations in the manufacturing sector to support their sustainability and help create safer and more productive workplaces.

A sizing survey conducted in the right way will enable better design and safer and healthier work places and practices. 


\section{References}

1. Veitch D, Caple D, Blewett V. Sizing up Australia: How Contemporary is the Anthropometric Data Australian Designers Use? Australia: Australian Government, Australian Safety and Compensation Council; 2009.

2. Veitch D, Fitzgerald C, Blewett V, Ward S, Shu C, Robinette K. Australian Body Sizing Survey:. Safe Work Australia, in press.

3. Veitch D, Blewett V, Caple D. Sizing up Australia: towards national sizing survey. Work IOS Press. 2012;41:4083-7.

4. Robinette KM, Vannier MW, Rioux M, Jones PRM. 3-D Surface Anthropometry: Review of Technologies. AGARD Advisory Report. 1997;329.

5. WEAR Members. TUDelft; 2013 [cited 201310 May]. Available from: http://wear.io.tudelft.nl/.

6. CODATA. International Council for Science: Committee on Data for Science and Technology CODATA, Task Group on Anthropometric Data and Engineering: CODATA; 2013 [cited 20135 May]. Available from: http://www.codata.org/taskgroups/TGanthro/.

7. TC2. 2013 [28 Septemeber 2013]. Available from: http://www.tc2.com/index 3dbodyscan.html.

8. Robinette $\mathrm{K}$, Daanen $\mathrm{H}$. Precision of the CAESAR scan-extracted measurements. Applied Ergonomics. 2006;37(3):251-386.

9. Veitch D. Where is the human waist? Definitions, manual compared to scanner measurements. 20th World Congress on Ergonomics — International Ergonomics Association (IEA); Recife Brazil 2012.

10.Cheng H, Robinette K. XML Based Networking Method for Connecting Distributed Anthropometric Databases Name. Data Science Journal. 2007; Volume 6(Supplement).

11. International Standards Organisation (ISO). ISO 7250-1 Basic human body measurements fo technological design. Part 1: Body measurement definitions and landmarks. Geneva: ISO; 2008. p. 25.

12.ISO. ISO/TR 7250-2 Basic human body measurements for technological design. Part 2: Statistical summaries of body measurments from individual ISO populations. Geneva: ISO; 2010. p. 53.

13.ISO. ISO 15535 General requirements for establishing anthropometric databases. Geneva: ISO; 2006. p. 20.

14.International Standards Organisation (ISO). ISO 20685 3-D scanning methodologies for internationally compatible anthropometric databases. Geneva: International Standards Organisation (ISO); 2010. p. 20.

15. International Standards Organisation (ISO). ISO 8559: Garment construction and anthropometric surveys - Body dimensions, International Standards Organisation: International Standards Organisation (ISO); 1989.

16. Mochimaru M, editor ISO Projects for Anthropometry. 1st Asian Workshop on 3D Body Scanning Technologies; 2012; Tokyo, Japan.

17. Ennis E, Robinette K. Standardization for the purpose of the comparison of international data. Introducing the WEAR Data Network (AMI and ARIS). WEAR World Engineering Anthropometry Resource; Adelaide, Australia 2011.

18.Kouchi M, Mochimaru M, Bradtmiller B, Daanen H, Li P, Nacher B, et al. Errors in landmarking and the evaluation of the accuracy of traditional and $3 D$ anthropometry. APPLIED ERGONOMICS 2011; 42:518-27.

19.Wuhrer S, Xi P, Shu C. Human Shape Correspondence with Automatically Predicted Landmarks. Machine Vision and Applications. 2013; 23:821-30.

20.Mellian SA, Ervin C, Robinette KM. Sizing Evaluation of Navy Women's Uniforms. Technical Report No 182, Navy Clothing and Textile Research Facility, Natick MA and AL-TR-1991-0116, Armstrong Laboratory, Air Force Systems Command, Wright-Patterson Air Force Base OH. 1990.

21.Zehner G. Prediction of Anthropometric Accommodation in Aircraft Cockpits. United States Air Force Research Laboratory. 2001. PubMed PMID: ADA399750.

22.Zehner G, Hudson JA. Body Size Accommodation in USAF Aircraft. United States Air Force Research Laboratory. 2002. PubMed PMID: ADA405598.

23. Robinette K, Blackwell S, Daanen H, Fleming, Boehmer M, Brill T, et al. Civilian American and European Surface Anthropometry Resource (CAESAR), Final Report, Volume I: Summary. AFRL-HE-WP-TR-2002-0169. 2002.

24.Robinette K. Body Scanner Standard Overview. ASTM D-13 55; Washington DC 2005.

25. Robinette K, Daanen HAM, editors. Lessons learned from CAESAR: a 3-D anthropometric survey. Proceedings of the XVth Triennial Congress of the International Ergonomics Association; 2003: Ergonomics in the Digital Age. 
26. Allen B, Curless B, Popvic Z. The space of human body shapes: reconstruction and parameterization from range scans. ACM SIGGRAPH 2003. 2003.

27. Hudson J, Zehner GF, Robinette KM. JSF CAESAR: Construction of a 3-D anthropometric sample for design and sizing of Joint Strike Fighter Pilot Clothing and Protective Equipment. AFRL-HE-WP-TR-2003-0142. 2003.

28.Ben Azouz Z, Rioux M, Shu C, Lepage R. Analysis of Human Shape Variation using Volumetric Techniques. Proc of 17th Annual Conference on Computer Animation and Social Agents; Geneva, Switzerland 2004.

29.Anguelov D, Srinivasan P, Koller D, Thrun S, Rodgers J, Davis J. SCAPE: shape completion and animation of people. ACM Transactions on Graphics. 2005; 24(3):408-16.

30. Allen B, Curless B, Popovic Z, Hertzmann A. Learning a correlated model of identity and pose-dependent body shape variation for real-time synthesis. ACM SIGGRAPH/Eurographics Symposium on Computer Animation. 2006:147-56.

31.Ben Azouz Z, Shu C, Mantel A. Automatic locating of anthropometric landmarks on 3D human models. International Conference on 3D Data Processing, Visualization and Transmission (3DPVT) 2006.

32.Veitch D, Robinette K. BioFidelic Apparel Manikins from CAESAR 3D Anthropometric Data. The International Ergonomics Association Congress,; Maastricht, The Netherlands 2006.

33.Xi P, Lee W-S, Shu C. Analysis of Segmented Human Body Scans. Graphics Interface Conference 2007. 2007:19-26.

34.Veitch D, Dawson R, Owen H, Leigh C. The Development of a Lifelike Breast Cancer Patient Simulator using Anthropometric Data. Ergonomics Australia. 2011; Special edition.

35.Wuhrer S, Shu C, Xi P. Landmark-Free Posture Invariant Human Shape Correspondence. Visual Computer. 2011; 27(9):843-52.

36. Wuhrer S, Shu C, Bose P. Automatically Creating Design Models from 3D Anthropometry Data. ASME Journal of Computing and Information Science in Engineering. 2012; 12(4). 